\title{
ON AN ASSUMPTION CONTAINED IN EUCLID'S PROOFS OF CERTAIN PROPOSITIONS IN HIS TIVELFTH BOOK
}

\author{
By J. Rose-Innes.
}

[Received March 11th, 1913.-Read April 24th, 1913.]

Ir is well known that Euclid in his proof of $V, 18$ made the assumption that, given any three quantities - the first two being of the same kindthere always exists a fourth proportional to them. Simson, who objectel to the proof as it stands, devised another free from the supposed blemish. The assumption reappears in Euclid in tive propositions of his Twelfth Book, viz., XII, 2, 5, 11, 12, 18, and Simson did not give any proof of these theorems removing the difficulty; nor has he put forward any grounds which entitle him to condone the want of rigour in the Twelfth Book while condemning it in the Fifth. As far as I know, none of the subsequent commentator's has cleared up the matter. It seems interesting, therefore, to enquire to what extent the above assumption is really necessary, and to examine how Euclid could have managed to do without it, had he entertained any objection to it, while adhering in other respects to his own methods and his own definitions. In other words, we might wish to know whether it is possible to make for the five theorems of the T'welfth Book the same kind of amendment that simson effected in V, 18.

After a careful examination as to the best way of removing the diffculty, I am led to believe that in the case of XII, 2 we can proceed most readily by making use of Stolz's theorem. This theorem tells us that in order to satisfy ourselves that four quantities, say $A, B, C, D$ are in pro. portion, we need only show that $p A>q B$, according as $p C<q D$; where $p A, p C$ are any equimultiples of $A$ and $C$, and $q B, q D$ are any independent equimultiples of $B$ and $D$. The remaining test contemplated by Euclid as to $p A=q B$, when $p C=q D$, need not be applied. (See Stolz, Vorlesungen über allgemeine Arithmetik, Pt. 1, p. 87, published in 1885.)

The proof of XII, 2 on these lines will now be given.

To show that any two circles have the same ratio as the squares on their diameters. 
Let $C_{1}$ and $C_{2}$ be the two circles ; take $n_{1} C_{1}, n_{2} C_{2}$ multiples of $C_{1}$ and $C_{3}$ respectively, and first suppose that $n_{1} C_{1}>n_{2} C_{2}$.
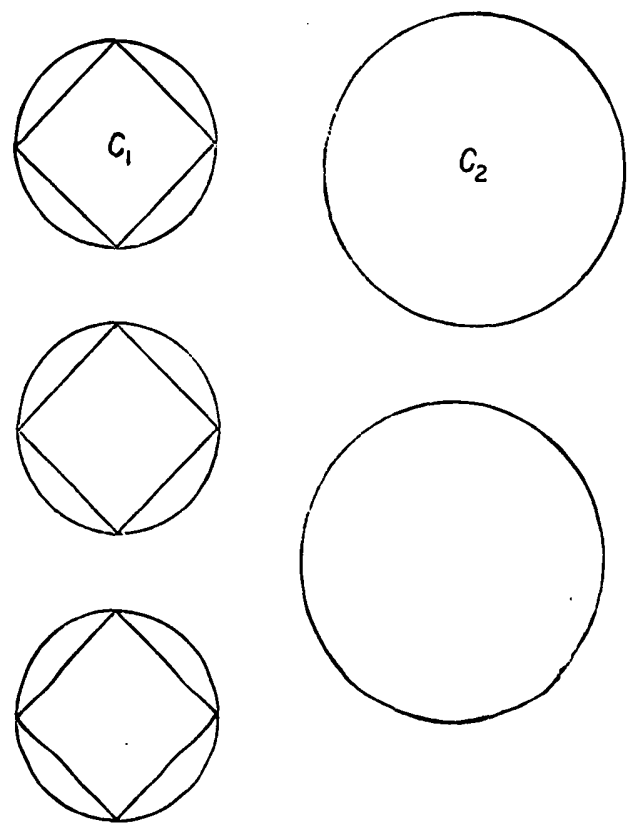

Inscribe a square in each of the circles equal to $C_{1}$; then just as in Euclid we may show that each square is greater than the half of the circle in which it is inscribed; and that on substituting for each square an inscribed regular polygon of double the number of sides we shall be removing from the segments left over from the squares more than their half. (See Euclid's proof ; Heath, Vol. III, pp. 371, 372.)

Thus, by starting with the inscribed squares and continually substituting inscribed regular polygons of double the number of sides, we shall at length leave some segments of the circles which will be together less than the excess by which $n_{1} C_{1}$ exceeds $n_{2} C_{2}(\mathrm{X}, 1)$.

Let $P_{1}$ denote the inscribed polygon when this stage has been reached; then

$$
n_{1} C_{1}-n_{1} P_{1}<n_{1} C_{1}-n_{2} C_{2},
$$

so that

$$
n_{1} P_{1}>n_{2} C_{2} \text {. }
$$

Let $P_{2}$ denote a polygon similar to $P_{1}$ but inscribed in $C_{2}$; then

$$
n_{2} C_{2}>n_{2} P_{2} \text {. }
$$

Hence

$$
n_{1} P_{1}>n_{2} P_{2} \text {. }
$$


But similar polygons inscribed in circles are to one another as the squares on the diameters (XII, 1). Therefore

$$
n_{1} S_{1}>n_{2} S_{2},
$$

where $S_{1}, S_{2}$ are the squares on the diameters of $C_{1}, C_{2}$ respectively (V, Def. 5).

Secondly, if $n_{1} C_{1}<n_{2} C_{2}$, we may show in a similar manner that

$$
n_{1} S_{1}<n_{2} S_{2} \text {. }
$$

By Stolz's theorem these two cases are sufficient to prove that $C_{1}, C_{2}$, $S_{1}, S_{2}$ are proportional.

An objection might be rrised to the above proof, viz., that Stolz's theorem does not actually occur in Euclid, and that consequently we have no right to use the theorem in a proof conducted professedly on Euclidean lines. To this we might urge in reply that though Stolz's theorem is not actually included in Euclid's Fifth Book, it can easily be proved by Euclidean methods (see Hill's Euclid, 2nd ed., p. 29); and we may therefore imagine that it has been proved and added to the Fifth Book just as Simson, for example, added his propositions $A-K$.

The remaining propositions referred to above, viz., XII, $5,11,12,18$, can all be proved by Stolz's theorem, without making use of Euclid's assumption, on much the same lines as XII, 2 in the proof just given. It seems worth mentioning that for three of the propositions, XII, 5, 11, 12, we can easily devise a proof by ordinary geometrical manipulation, which shall avoid Euclid's assumption, and yet be independent of Stolz's theorem. For XII, 12, though not for XII, 5 and 11, the alternative proof is decidedly the simpler. 